\title{
Correction of Mandibular Retrognathia and Laterognathia by Distraction Osteogenesis: Follow up of 5 cases
}

\author{
Dogan Dolanmaza \\ Ali Ihya Karaman ${ }^{\mathrm{b}}$ \\ Hakan Gurcan Gurelc \\ Abdullah Kalaycid \\ Hasan Kucukkolbasie \\ Serdar Usumez
}

\section{ABSTRACT}

This article evaluates the use of distraction osteogenesis in the treatment of mandibular retrognathia and laterognathia and the long term treatment results of the patients treated with this technique. The procedure was carried out in 5 subjects ( 3 males and 2 females, mean age 18.4 years) aged between 14 years and 27 years. In patients treated with bilateral mandibular distraction, it was observed that the ANB angle decreased by a mean of $5^{\circ}$, the mandibular corpus length increased by a mean of $14.5 \mathrm{~mm}$ and the overjet decreased by a mean of $12.2 \mathrm{~mm}$ after treatment. In patients treated with unilateral mandibular distraction, a mean of $3.5^{\circ}$ reduction was achieved in ANB angle, the mandibular corpus length increased by a mean of $5.5 \mathrm{~mm}$ and a mean of $7 \mathrm{~mm}$ correction was achieved in relation to craniofacial midline with treatment. One of these patients showed an increase of $10 \mathrm{~mm}$ in ramus height on the affected side and a decrease of $5^{\circ}$ in gonial angle whereas the other one showed an increase of $12.5^{\circ}$ in gonial angle and an increase of $11 \mathrm{~mm}$ in ramus height on the affected side after treatment. The most significant long term relapse was observed in one of the patients treated with bilateral mandibular distraction. Long term relapse seen in the rest of the patients was within clinically acceptable limits. It can be concluded that distraction of the deformed mandible is a feasible and effective technique for treating mandibular retrognathia and laterognathia. However, it must be borne in mind that accurate placement of the distractors and determining the correct distraction vector are crucial factors that have an influence on long term clinical success. (Eur J Dent 2009;3:335-342)

Key words: Distraction osteogenesis; Mandibular retrognathia; Laterognathia; Orthodontic treatment.

a Associate Professor, Selcuk University, Faculty of Dentistry, Department of Oral and Maxillofacial Surgery, Konya, Turkey.

b Professor, Kocaeli University, Faculty of Dentistry, Department of Orthodontics, Kocaeli, Turkey.

Research Fellow, GATA Military Research and Training Hospital, Dental Clinic, Section of Orthodontics, Istanbul, Turkey.

d Assistant professor, Selcuk University, Faculty of Dentistry, Department of Oral and Maxillofacial Surgery, Konya, Turkey.
Assistant professor, Selcuk University, Faculty of Dentistry, Department of Oral and Maxillofacial Surgery, Konya, Turkey.

Associate Professor, Gaziantep University, Faculty of Dentistry, Department of Orthodontics, Gaziantep, Turkey.

- Corresponding author: Hakan Gurcan Gurel GATA Military Research and Training Hospital Dental Clinic, Section of Orthodontics Haydarpasa, Istanbul, Turkey. E-mail: hggurelahotmail.com 


\section{INTRODUCTION}

Distraction osteogenesis has become a widely accepted procedure in orthopedics and has been applied to treat the skeletal deformities and severe bony defects in the craniofacial complex. ${ }^{1-8}$ With this procedure, bone volume can be increased by gradual traction of a fracture callus formed between osteotomized bony segments. ${ }^{8-10}$ Bone lengthening by osteotomy and distraction osteogenesis of long bones was first described in 1905 by Codvilla and popularized by Ilizarov. Mandibular lengthening by gradual distraction was reported in 1973 by Synder et al who used an extraoral device in a canine study; new bone formation at the elongated site was demonstrated later by Karp et al. In 1992, McCarthy et al successfully elongated the mandible by up to $24 \mathrm{~mm} .7,8,11,12$ Distraction osteogenesis is particularly useful for treating cases of severe bony hypoplasia where the surgical movement required to correct the malocclusion is outside the range predictably achievable with routine orthognathic surgery techniques. $8,10,13$

In patients for whom only mandibular deficiencies need to be corrected surgically, bilateral sagittal split osteotomy (BSSO) is the most common procedure. Intra-oral distraction osteogenesis during orthodontic treatment as a solution for a Class II malocclusion has been proposed as an alternative to BSSO. ${ }^{14-19}$ Mandibular distraction is becoming a prevalent surgical treatment for retrognathia and asymmetry, and many reports have demonstrated that this technique provides great clinical benefits for mandibular deficiency and other craniofacial deformities.7,8,20,21 One of the shortcomings of distraction osteogenesis, however, is that accurate positioning of the proximal segment can be difficult to achieve either because of an inaccurate displacement vector or because of an unpredictable soft tissue influence on the immature regenerate. It has been shown in an animal model and in clinical case reports that postdistraction regenerate can be molded by external forces. ${ }^{13,22-26}$

van Stiejen et al studied the complications in bilateral mandibular distraction osteogenesis and recorded a total of 28 complications in their study sample of 70 patients. They reported osteotomy related complication in 1 patient, technique and/ or device-related complications in 10 patients, infection in 5 patients, prolonged sensory loss in the distribution of the alveolar nerve in 3 patients, compliance related complications in 6 patients and condylar problems in 3 patients. ${ }^{27}$

In this paper, the results of mandibular lengthening and correction of mandibular asymmetry by distraction osteogenesis are demonstrated.

\section{SUBJECTS AND METHODS}

The procedure was carried out in 5 subjects ( 3 males and 2 females, mean age 18.4 years) aged between 14 years and 27 years. The orthodontic and surgical treatment of the subjects was performed at the same center. Written informed consent was obtained from the parent or patient before treatment. The detailed information on subjects is shown in Table 1.

Two patients had mandibular asymmetry resulting from odontogenic infection in one and from trauma in the other and the other three had sagittal mandibular deficiency. Of these three patients, one had a history of trauma during early childhood and in the other two the aetiology was unclear. One of these three patients underwent mandibular advancement surgery with BSSO. However, because of relapse seen after treatment, a decision was made to perform mandibular advancement by distraction osteogenesis. Three of five patients underwent bilateral mandibular distraction and in the remaining two patients, unilateral mandibular distraction osteogenesis was performed to correct mandibular asymmetry. Of these 2 patients, one underwent surgically assisted rapid maxillary expansion prior to unilateral mandibular distraction and the other one received surgery for the treatment of the ankylosis of the temporomandibular joint. However, the asymmetry remained.

All five patients had pre-and post-operative orthodontic treatment with fixed appliances and distraction osteogenesis surgery was carried out under general anesthesia in all cases. Lateral and posteroanterior cephalometric and panoramic radiographs were taken from all patients on three different occasions (before treatment, after treatment and during follow-up period). The landmarks and planes used in cephalometric analysis are shown in Figure 1. Further surgical procedure included genioplasty in three patients.

In patients 4 and 5, a complete oblique osteotomy of the ascending ramus was made through 
an intraoral approach protecting inferior alveolar nerve. Due to difficulty experienced in placing the distractors intraorally, the distractors were placed after exposing the ramus through a $2.5 \mathrm{~cm}$ long extra-oral submandibular incision. Prior to completion of the osteotomy, distractor (VasquezDiner type intra-oral distractor, Leibinger, Germany I was adapted to the bone surface. An effort was made to place the distractor perpendicular to the osteotomy line. After a latency period of 1 week, active distraction was started at a rate of 0.5 $\mathrm{mm}$ twice per day.

In patients 2 and 3, intra-oral distractors (Medartis, Modus MDO 2.0, Basel, Switzerland) were used and an extra-oral distractor (Molina bi-directional extraoral mandibular distractor, KLS Martin, USA) was used in patient 1. The intra-oral distractor was placed as parallel as possible to the maxillary occlusal plane after performing a vertical osteotomy in the lower retromolar region through an intra-oral approach and the extra-oral

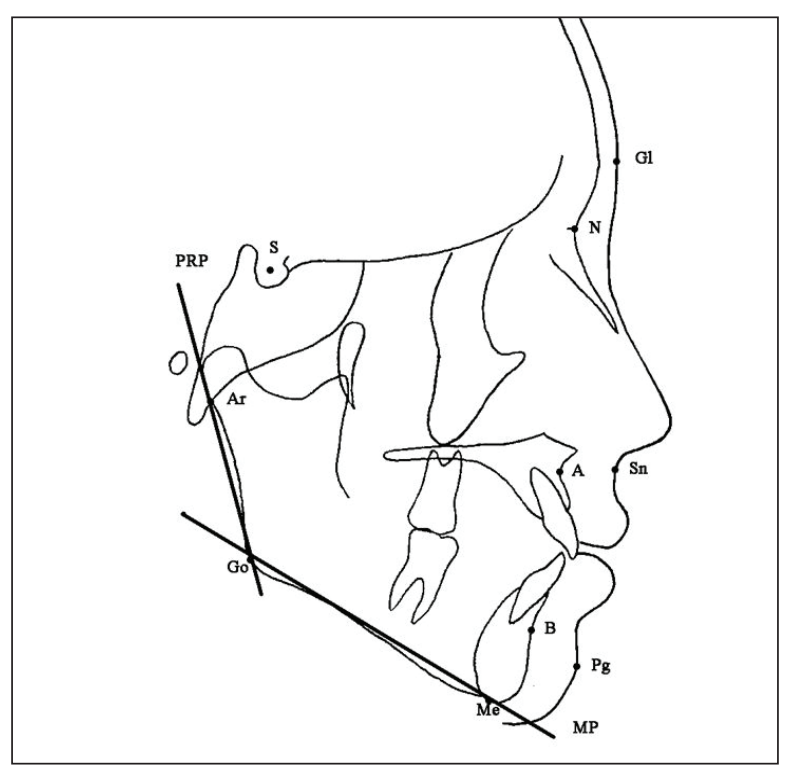

Figure 1. Landmarks and planes used in lateral cephalometric analysis. distractor was placed in the ascending ramus after performing an oblique osteotomy through an intra-oral approach. The fixation pins of the extraoral distractor were attached to the basal mandibular bone transcutaneously close to the posterior border of ramus above the osteotomy line. Following a latency period of 1 week, activation was started at a rate of $0.5 \mathrm{~mm}$ twice per day at the vertical rod of the distractor (in patient 1), then continued at the horizontal rod until the sagittal mandibular deficiency was resolved. Once desired mandibular lengthening was achieved, the fixation screw was loosened and then moulding of the regenerate was carried out using anterior heavy elastics to obtain a stable occlusion. On the completion of the moulding procedure, the consolidation period started and lasted 12 weeks.

\section{RESULTS}

In patient 4 , the distraction rod fractured towards the end of the distraction procedure. The rod was then removed and the distractor was left in place for 3 months for the consolidation of the regenerate. Afterwards, the broken distractor was removed and replaced with another intra-oral distractor (Guerrero-Bell type intra-oral distractor, Leibinger, Germanyl following a vertical osteotomy made in the mandibular corpus.

In all patients, paresthesia developed on the related side(s) following surgery and intensified during distraction period. However, it disappeared gradually in the long term. Genioplasty was required in some patients as mandibular asymmetry could no further be corrected by distraction osteogenesis once a CL I canine relationship was established bilaterally.

In patients 1,2 and 3, it was observed that the ANB angle decreased by a mean of $5^{\circ}$, the mandibular corpus length increased by a mean of 14.5

Table 1. Clinical features of the patients.

\begin{tabular}{|c|c|c|c|c|c|}
\hline Patient & Age & Sex & Type of Distracion & Genioplasty & Follow-up period \\
\hline 1 & 14 & $\mathrm{~F}$ & Bilateral extra-oral & Yes & 4.1 years \\
\hline 2 & 17 & M & Bilateral intra-oral & No & 3.8 years \\
\hline 3 & 18.3 & $\mathrm{~F}$ & Bilateral intra-oral & Yes & 5.3 years \\
\hline 4 & 27 & M & Unilateral intra-oral & No & 4 years \\
\hline 5 & 16.3 & M & Unilateral intra-oral & Yes & 4.2 years \\
\hline
\end{tabular}


$\mathrm{mm}$ and the overjet decreased by a mean of 12.2 $\mathrm{mm}$ after treatment. Ramus height increased by $3 \mathrm{~mm}$ in one patient only. Increase in gonial angle was observed in post-treatment records in all 3 patients except one who later exhibited relapse in follow-up records. Moreover, a significant increase in convexity angle was observed on the completion of treatment in all 3 patients. Of all these 3 patients, patient 3 exhibited the most significant relapse in ANB angle, gonial angle, horizontal overjet and convexity angle in follow up records.

In patients 4 and 5 , a mean of $3.5^{\circ}$ decrease was observed in ANB angle and the mandibular corpus length increased by a mean of $5.5 \mathrm{~mm}$ after treatment. One of these patients showed an increase of $10 \mathrm{~mm}$ in ramus height on the affected side and a decrease of $5^{\circ}$ in gonial angle whereas the other one showed an increase of $12.5^{\circ}$ in gonial angle

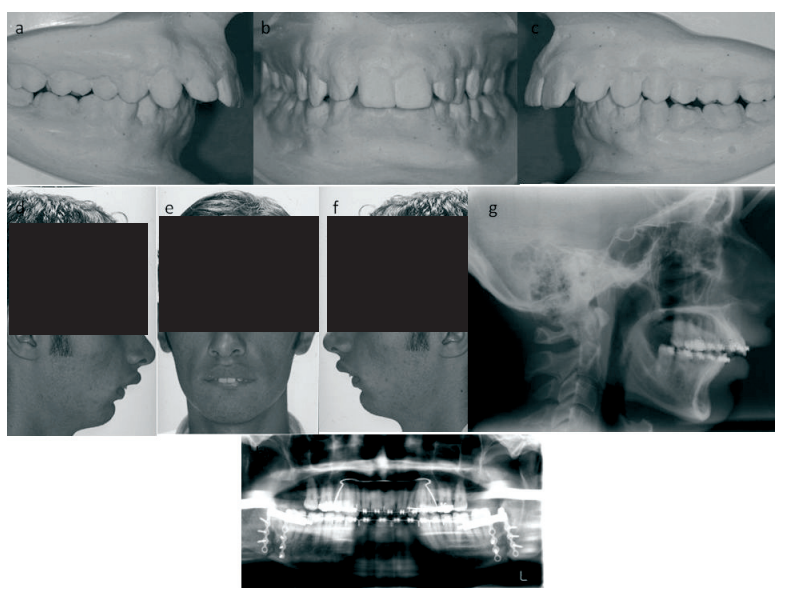

Figure 2. (a-c) Pre-treatment study casts, (d-f) pre-treatment extra-oral photographs, (g) pre-distraction lateral cephalometric radiograph and (h) pre-distraction panoramic radiograph of Patient 2.

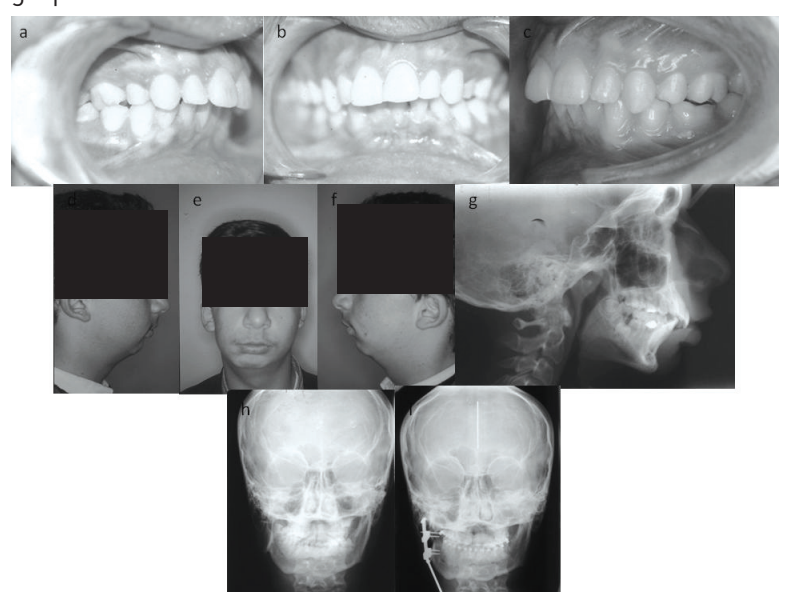

Figure 4. Pre-treatment (a-c) intra-oral photographs, (d-f) extra-oral photographs, (g) lateral cephalometric radiograph, (h) PA cephalometric radiograph and (i) pre-distraction PA cephalometric radiograph of Patient 5. and an increase of $11 \mathrm{~mm}$ in ramus height on the affected side after treatment. In both patients the horizontal overjet was reduced with treatment and the convexity angle became more obtuse. A mean of $7 \mathrm{~mm}$ correction was achieved in relation to craniofacial midline. Follow up records indicated $1 \mathrm{~mm}$ of relapse in horizontal overjet in patient 4 and $0.2 \mathrm{~mm}$ of relapse in horizontal overjet and $1^{\circ}$ of relapse in convexity angle in patient 5 . The results of lateral cephalometric analysis are shown in Table 2. Pre- and post-treatment photographs and radiographs along with pre-treatment study casts of patient 2 are shown in Figures 2 and 3 . Pre-and post- treatment photographs and radiographs of patient 5 are shown in Figures 4 and 5.

\section{DISCUSSION}

Bilateral sagittal split osteotomy (BSSO) and distraction osteogenesis are the most common

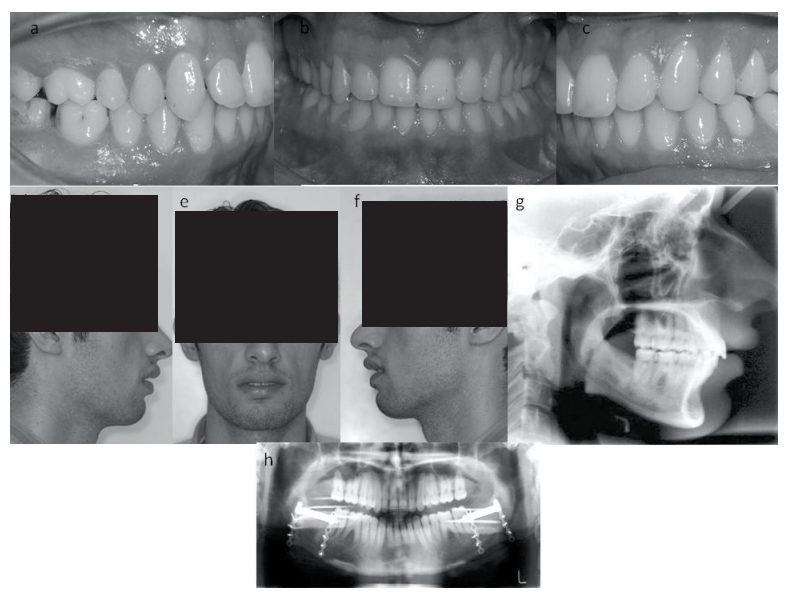

Figure 3. Post-treatment (a-c) intra-oral photographs, (d-f) extra-oral photographs, (g) lateral cephalometric radiograph and (h) panoramic radiograph of Patient 2.

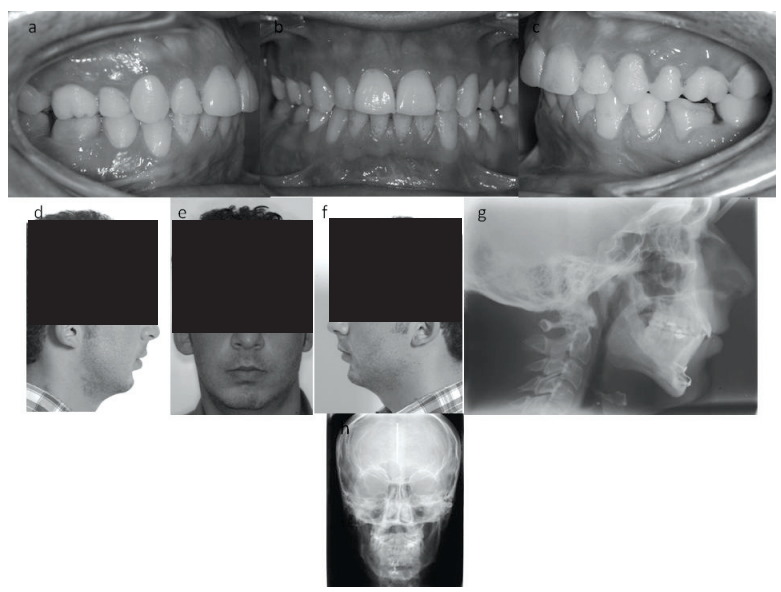

Figure 5. Post-treatment (a-c) intra-oral photographs, (d-f) extra-oral photographs, (g) lateral cephalometric radiograph and (h) PA cephalometric radiograph of Patient 5. 
techniques currently applied to surgically correct mandibular deformities. Although randomized clinical trials are lacking, some support was found in the literature for distraction osteogenesis having advantages over BSSO in the surgical treatment of low and normal mandibular plane angle patients needing greater advancement Igreater than $7 \mathrm{~mm}$ ). In all other mandibular retrognathia patients the treatment outcomes of distraction osteogenesis and BSSO seemed to be comparable. ${ }^{28}$ Differential growth and conventional orthognathic procedures become more difficult and less predictable when correcting severe mandibular deficiencies requiring lengthening of the mandible more than $8-10 \mathrm{~mm} .{ }^{29}$ The primary advantage claimed in connection with distraction osteogenesis is that it allows major reshaping of the facial bones without bone grafts or jaw wiring. It is believed that distraction osteogenesis may be safer than other methods of facial reconstruction, since it can involve less blood loss and a lower risk of infection. ${ }^{30}$ Moreover, reports on patients with cleft palate have suggested that maxillary advancements achieved by distraction are more stable than the advancements achieved with orthognathic surgery. ${ }^{31,32}$

The specially fabricated hardware used for the distraction process can be internal or external. Advantages of external devices include ease of placement and removal. In addition, some ex- ternal devices allow multi-dimensional control. External devices, however, are very conspicuous and are more likely to cause traction scars than internal devices. Although intra-oral distractors are known to have some advantages (i.e. no facial scar, better tolerance by the patient), they are only unidirectional and require a subsequent surgical procedure for their removal. ${ }^{33}$ We observed ulcerative lesions around the distractor pins particularly during the consolidation period and postoperative facial scar in patient 1 . Our experience leads us to think that the placement of intra-oral distractors in desired locations is associated with the experience of the surgeon. For this reason, the surgical procedure for the placement of intra-oral distractors took longer than that for extra-oral distractors.

The nature of distraction osteogenesis is well suited for stretching of the pterygomasseteric sling, which is not easily overcome by conventional procedures. Pterygoid muscle usually does not adapt to the elongation of ramus. However, during distraction osteogenesis, active histiogenesis occurs in different tissues including gingiva, blood vessels, ligaments, cartilage, muscles and nerves. These adaptive changes in the soft tissues decrease the relapse risk and allow the treatment of severe facial deformities. ${ }^{34,35}$ Although we observed a significant increase in ramus height on the affected side in patients 4 and 5 . We did not

Table 2. Results of lateral cephalometric analysis.

\begin{tabular}{|c|c|c|c|c|c|c|c|c|c|c|c|c|c|c|c|}
\hline \multirow{2}{*}{$\begin{array}{l}\text { Patients } \\
\text { Stages }\end{array}$} & \multicolumn{3}{|c|}{1} & \multicolumn{3}{|c|}{2} & \multicolumn{3}{|c|}{3} & \multicolumn{3}{|c|}{4} & \multicolumn{3}{|c|}{5} \\
\hline & PreT & РoT & FU & PreT & РoT & $\mathrm{FU}$ & PreT & Рот & FU & PreT & PoT & $\mathrm{FU}$ & PreT & Рот & $\mathrm{FU}$ \\
\hline SNA (deg) & 75.5 & 76 & 76 & 84 & 84 & 84 & 77.5 & 78 & 78 & 78 & 78 & 78 & 75 & 76 & 76 \\
\hline SNB (deg) & 67 & 73 & 73 & 76 & 80 & 80 & 69 & 75 & 74 & 74 & 75 & 75 & 63 & 70 & 70 \\
\hline ANB (deg) & 8.5 & 3 & 3 & 8 & 4 & 4 & 8.5 & 3 & 4 & 4 & 3 & 3 & 12 & 6 & 6 \\
\hline \multicolumn{16}{|l|}{ Corpus length, } \\
\hline Me-Go (mm) & 53 & 65 & 65 & 72 & 87 & 87 & 65.5 & 83 & 83 & 65 & 70 & 70 & 55 & 61 & 61 \\
\hline Ramus height, & & & & & & & & & & L 49 & L 59 & L 59 & L 80 & L 80 & L 80 \\
\hline Go-Ar, Go-Co* (mm) & 39 & 42 & 42 & 56 & 56 & 56 & 36 & 36 & 36 & R 75 & R 75 & R 75 & $\mathrm{R} 60$ & R 71 & R 71 \\
\hline \multicolumn{16}{|l|}{ Gonial angle } \\
\hline (PRP-MP) (deg) & 121 & 154 & 153 & 111 & 113 & 113 & 133 & 125 & 128 & 129 & 124 & 124 & 118.5 & 131 & 131 \\
\hline \multicolumn{16}{|l|}{ Horizontal } \\
\hline overjet (mm) & 17.5 & 5 & 5 & 18 & 4 & 4.5 & 13 & 3 & 4 & 12 & 4 & 5 & 9 & 3.8 & 4 \\
\hline \multicolumn{16}{|l|}{ Convexity angle } \\
\hline (GL-Sn-Pg) (deg) & 147.5 & 155 & 155 & 144 & 159 & 159 & 154 & 163.5 & 162 & 165 & 170 & 170 & 142.5 & 156 & 155 \\
\hline
\end{tabular}

* Patients with laterognathia

Deg: Degree; PreT: Pre-treatment; PoT: Post-treatment; FU: Follow-up; L: Left ramus; R: Right ramus. 
observe a significant increase in ramus length in patient 1 in whom the vertical component of the external distractor was activated. In our opinion, the activation did not translate exactly to the desired increase in ramus length due to angular alterations performed during distraction.

Patient 3 developed anterior open bite during distraction as a result of misplacement of the distractor and reduced vector control. Anterior open bite in this patient was corrected by moulding of the regenerate using intermaxillary elastics for 10 days following the removal of the distraction cylinders from the distractors in the second week of the consolidation period. However, follow up records taken 5.3 years after the completion of treatment indicated relapse of the anterior open bite. Gateño et al suggested that computer assisted surgical planning and modeling could be helpful in determining the correct distraction vector and in accurate placement of the distractors. ${ }^{36}$ In our opinion, the long term relapse observed in Patient 3 could have been avoided or its rate could have been decreased through the use of computer assisted surgical planning and modeling. The unilateral posterior open bite seen in patients 4 and 5 during distraction was corrected by orthodontic extrusion of the posterior teeth using elastic traction. No anterior open bite was seen in patient 2 in whom an effort was made to place the distractors parallel to the maxillary occlusal plane. We believe that gonial angle increase in patients 1,3 and 5 was caused by the downward relocation of menton point as a result of genioplasty. Although genioplasty was deemed indicated for the patients 2 and 4, it was not performed as the patients were satisfied with the treatment result.

A possible advantage of distraction is its effect on the inferior alveolar nerve. Makarov et al suggested that if acute nerve injury is avoided with surgery, then up to $10 \mathrm{~mm}$ of distraction of the mandible would appear to produce minimal effects on inferior alveolar nerve function. ${ }^{37}$ In all patients, paresthesia developed on the related side(s) following surgery and intensified during distraction period. However, it diminished gradually after the treatment and was not observed in follow-up examinations.

Marked changes occurred in the position and shape of the lower jaw and in the occlusion as a result of mandibular distraction. Even though the ramus height remained unchanged in patients 2 and
3, significant amount of lengthening was achieved in mandibular corpus in patients 1,2 and 3. An increase in ramus height was observed in patients 4 and 5 on the affected side. Anterior movement of the lower jaw resulted in sagittal improvement of the prominence of the chin point. However in patients 1,3 and 5, genioplasty was deemed necessary as required chin prominence could not be achieved by distraction alone due to limitation imposed by post-distraction dental occlusion.

\section{CONCLUSIONS}

It can be concluded that distraction of the deformed mandible is a feasible and effective technique for treating mandibular retrognathia and laterognathia and that long term relapse is within acceptable limits. However, it must be borne in mind that accurate placement of the distractors and determining the correct distraction vector are crucial factors that have an influence on long term clinical success.

\section{REFERENCES}

1. Paley D, Catagni MA, Argnani F, Villa A, Benedetti GB, Cattaneo R. Illizarov treatment of tibial nonunions with bone loss. Clin Orthop Relat Res 1989;241:146-165.

2. Samchukov ML, Ezaki M, Tucker WF, Ross JD. Restoration of apposition function in residual hand by Illizarov method: a case report. Bull Hosp Jt Dis Orthop Inst 1993;53:13-16.

3. Cope JB, Harper RH. Significant mandibular deficiency treated by osteodistraction: an alternative to traditional orthognathic surgery. World J Orthod 1999;5:25-34.

4. Bengi AO, Gürton AU, Okcu KM, Aydintug YS. Premaxillary distraction osteogenesis with an individual tooth-borne appliance. Angle Orthod 2004;74:420-431.

5. Sayin S, Bengi AO, Gurton AU, Ortakoglu K. Rapid canine distalization using distraction of periodontal ligament: a preliminary clinical validation of the original technique. Angle Orthod 2004;74:304-315.

6. Stucki MC, Cormick SU. Reconstruction of the mandibular condyle using transport distraction osteogenesis. J Craniofac Surg 1997;8:48-52.

7. Snyder CC, Levine GA, Swanson HM, Browne EZ Jr. Mandibular lengthening by gradual distraction: preliminary report. Plast Reconstr Surg 1973;51:506.

8. McCarthy JG, Schreiber J, Karp N, Thorne CH, Grayson BH. Lengthening the human mandible by gradual distraction. Plast Reconstr Surg 1992;89:1-8. 
9. Cope JB, Samchukov ML. Mineralization dynamics of regenerate bone during mandibular osteodistraction. J Maxillofac Surg 2001;30:234-242.

10. Hopper RA, Altug AT, Grayson BH, Barillas I, Sato Y, Cutting CB, McCarthy JG. Cephalometric analysis of the consolidation phase following bilateral pediatric mandibular distraction. Cleft Palate Craniofac J 2003;40:233-240.

11. Ilizarov GA. The principles of the Ilizarov method. Bull Hosp Joint Dis Orthop Inst 1988;48:1-11.

12. Karp NS, Schreider JS, Thorne CH, McCarthy JG. Membranous bone lengthening: a serial histologic study. Ann Plast Surg 1992;29:2-7.

13. Van Sickels JE. Distraction osteogenesis versus orthognathic surgery. Am J Orthod Dentofacial Orthop 2000;118:482484.

14. Van Sickels JE, Richardson DA. Stability of orthognathic surgery: a review of rigid fixation. Br J Oral Maxillofac Surg $1996 ; 34: 279-285$.

15. van Strijen PJ, Perdijk FB, Becking AG, Breuning KH. Distraction osteogenesis for mandibular advancement. Int $J$ Oral Maxillofac Surg 2000;29:81-85.

16. van Strijen PJ, Perdijk FBT, Breuning KH. Osteo-distraction in Class II deficiencies, the treatment of choice? 2nd International Congress on Cranial and Facial Bone Distraction Processes Bologna, Italy: Mondruzzi. 1999; 259-263.

17. Breuning $\mathrm{KH}$, van Strijen PJ. Orthodontic indications for mandibular distraction. In: Samchukov ML, Cope JL, Cheraskhin AM, editors. Craniofacial distraction osteogenesis St. Louis: Mosby; 2001; 278-285.

18. Mommaerts MY. Mandibular distraction with a dynamic osteosynthesis system (MD-DOS). J Craniomaxillofac Surg 1996;24(suppl 1):7.

19. Razdolsky Y, Dressner S, El-Bialy T. Clinical application of ROD intraoral custom distraction devices. In: Samchukov ML, Cope JL, Cheraskhin AM, editors. Craniofacial distraction osteogenesis St. Louis: Mosby; 2001; 269-277.

20. Aronson J. Biological and clinical evaluation of distraction histogenesis. Clin Orthop 1994;301:2-3.

21. Tavakoli K, Stewart KJ, Poole MD. Distraction osteogenesis in craniofacial surgery: a review. Ann Plast Surg 1998;40:8899.

22. Gosain AK. Plastic Surgery Educational Foundation DATA Committee. Distraction osteogenesis of the craniofacial skeleton safety and efficacy report. Plast Reconstr Surg $2001 ; 17: 278-280$

23. Luchs JS, Stelnicki EJ, Rowe NM, Naijher NS, Grayson $\mathrm{BH}, \mathrm{McC}$ arthy JG. Molding of the regenerate in mandibular distraction: part 1: laboratory study. J Craniofac Surg 2002; 13:205-210.
24. Kunz C, Hammer B, Prein J. Manipulation of callus after linear distraction: a "lifeboat" or an alternative to multivectoral distraction osteogenesis of the mandible? Plast Reconstr Surg 2000;105:674-679.

25. McCarthy JG, Hopper RA, Hollier LH, Peltomaki T, Katzen $\mathrm{T}$, Grayson $\mathrm{BH}$. Molding of the regenerate in mandibular distraction: clinical experience. Plast Reconstr Surg 2003; 112:1239-1246.

26. Fritz MA, Sidman JD. Distraction osteogenesis of the mandible. Curr Opin Otolaryngol Head Neck Surg 2004;12: 513518.

27. van Strijen PJ, Breuning KH, Becking AG, Perdijk FB, Tuinzing DB. Complications in bilateral mandibular distraction osteogenesis using internal devices. Oral Surg Oral Med Oral Pathol Oral Radiol Endod 2003;96:392-397.

28. Schreuder WH, Jansma J, Bierman MW, Vissink A. Distraction osteogenesis versus bilateral sagittal split osteotomy for advancement of the retrognathic mandible: a review of the literature. Int J Oral Maxillofac Surg 2007;2:103-110.

29. American Association of Oral and Maxillofacial Surgeons (AAOMS). Distraction osteogenesis. Statements by the American Association of Oral and Maxillofacial Surgeons Concerning the Management of Selected Clinical Conditions and Associated Clinical Procedures Rosemont, IL: AAOMS; 2003.

30. Fujimori Y, Ueda K, Oba S. Additional distraction osteogenesis after conventional fronto-orbital advancement. J Craniofac Surg 2005;6:1064-1069.

31. Ko EW, Figueroa AA, Guyette TW, Polley JW, Law WR. Velopharyngeal changes after maxillary advancement in cleft patients with distraction osteogenesis using a rigid external distraction device: a 1-year cephalometric follow-up. $J$ Craniofac Surg 1999;312-320.

32. Figueroa AA, Polley JW. Management of severe cleft maxillary deficiency with distraction osteogenesis: procedure and results. Am J Orthod Dentofacial Orthop 1999;115:1-12.

33. Maull DJ. Review of devices for distraction osteogenesis of the craniofacial complex. Semin Orthod 1999;1:64-73.

34. Hawkinson RT: Retrognathia correction by means of an arcing osteotomy in the ascending ramus. J Prosthet Dent 1968;20:77-86.

35. Liou EJ, Huang CS. Rapid canine retraction through distraction of the periodontal ligament. Am J Orthod Dentofacial Orthop 1998;114:372-382.

36. Gateño J, Teichgraeber JF, Aguilar E. Computer planning for distraction osteogenesis. Plast Reconstr Surg 2000;105:873-882. 
$\square$ Correction of mandibular retrognathia and laterognathia by distraction osteogenesis

37. Makarov MR, Harper RP, Cope JB, Samchukov ML. Evaluation of inferior alveolar nerve function during distraction osteogenesis in the dog. J Oral Maxillofac Surg 1998;56:1417-1423.

European Journal of Dentistry 